\title{
SISTEMA DE GESTÃO HOSPITALAR: UM ESTUDO DESCRITIVO DA SATISFAÇÃO DOS USUÁRIOS ${ }^{1}$
}

\section{HOSPITAL MANAGEMENT SYSTEM: A DESCRIPTIVESTUDY OF SATISFACTION OF USERS}

\section{HOSPITAL DEL SISTEMA DE GESTIÓN: UN ESTUDIO DESCRIPTIVO DE SATISFACCIÓN DE USUARIOS}

Sheila de Lima Cunha Dantas

Universidade Federal do Rio Grande do Norte - UFRN

sheilalc@gmail.com

\author{
Fernando Antonio de Melo Pereira \\ Universidade de São Paulo - USP \\ fernandopcmm@hotmail.com
}

\section{Resumo:}

Esta pesquisa tem como objetivo medir o nível de satisfação dos usuários quanto ao sistema de gestão hospitalar utilizado em um hospital de Natal/RN. Para tanto foi realizada uma pesquisa quantitativa, pelo método survey, realizada a partir da adaptação dos modelos de Ely (2010) e Wu e Wang (2007). Através da Análise Fatorial Exploratória (AFE) foi possível avaliar as variáveis medidas no instrumento de pesquisa, e através da Análise de Regressão chegou-se a um modelo final contendo três dimensões como fatores influenciadores da satisfação dos usuários com o sistema na organização objeto do estudo, são elas: confiança, tempo de resposta e envolvimento, sendo esta a principal colaboração desta pesquisa. A análise das evidências empíricas revelou que os usuários de forma geral encontram-se satisfeitos com o sistema e também foi possível apontar um conjunto de reflexões que podem contribuir para o aperfeiçoamento no uso do sistema hospitalar pesquisado, bem como para enriquecimento no meio acadêmico sobre o tema.

Palavras chave: Sistema de Informação (SI). Avaliação do SI. Satisfação do Usuário.

\begin{abstract}
:
This research aims to measure the level of users satisfaction about the hospital management system used in a hospital of Natal / RN city. For this purpose it was performed a quantitative research, using the method Survey, starting from the adaptation model of Ely (2010) and Wu and Wang (2007). Through Exploratory Factor Analysis was possible to validate the variables measured in the survey instrument, and by the regression analysis came to a final model containing three dimensions as influencing factors of user satisfaction with the system in the organization study object, they are: trust, response time and involvement, being this the main contribution of this research. The analysis of the empirical evidence revealed that users in general are satisfied with the system and it was also possible to present a set of reflections that can contribute to the improvement in the searched hospital system, as well as to knowledge enrichment in the mid of academic environment about the subject.

Key Words: Information System (IS). Evaluation of IS. User Satisfaction.
\end{abstract}

\section{Resumen}

Esta investigación tiene como objetivo medir el nivel de satisfacción de los usuarios en relación con el sistema de gestión del hospital se utiliza en un hospital de Natal / RN. Para esta investigación cuantitativa, el método de encuesta, hecha a partir de la adaptación de los modelos de Ely (2010) y se llevó a cabo Wu y Wang ( 2007 ). A través de un análisis factorial exploratorio (EPT) fue posible evaluar las variables medidas en el instrumento de la encuesta , y mediante el análisis de regresión que hemos llegado a un modelo final que contiene tres dimensiones como factores de satisfacción de los usuarios influir con el objeto del sistema en la organización del estudio Ellos son: fiabilidad, tiempo de respuesta y la participación , que es la principal aportación de esta

\footnotetext{
${ }^{1}$ Submetido em 19 de setembro de 2013. Aceito em 04 de junho de 2013. O artigo foi avaliado segundo o processo de duplo anonimato além de ser avaliado pelo editor. Editores responsáveis: Márcio Augusto Gonçalves e Lucas Maia dos Santos. Reprodução parcial ou total e trabalhos derivativos permitidos com a citação apropriada da fonte.
} 
investigación. El análisis de la evidencia empírica revela que los usuarios en general están satisfechos con el sistema y también fue posible identificar un conjunto de reflexiones que puedan contribuir a la mejora en el uso del sistema del hospital investigado y para el enriquecimiento en el ámbito académico sobre tema.

Palabras clave : Sistema de Información (SI ). Evaluación de la SI . Satisfacción de los Usuarios .

\section{INTRODUÇÃO}

Nas organizações, gerenciar as informações é fator primordial para tomada de decisão e integração dos processos. Os ERPs (Enterprise Resource Planning) e demais sistemas de informação dependem do uso eficiente dos usuários internos dos sistemas de gestão, pois serão eles os responsáveis por toda informação processada e armazenada da organização, e com a informação adequada à empresa poderá atingir suas metas e conquistar a liderança de mercado.

Para Pressman (1995) a qualidade de software é uma combinação complexa de fatores que variarão de acordo com diferentes aplicações e clientes que as solicitam. Não há dúvida que a qualidade de um software esta ligada à capacidade do sistema aplicativo atender as necessidades de quem o utiliza. Para Dias (1993) não existe uma medida objetiva e direta para medir a eficácia de um sistema de informação. Em geral, ela é avaliada pela capacidade do sistema desenvolvido apoiar os objetivos da empresa, segundo a percepção dos usuários do sistema.

Diversas pesquisas buscam compreender como o índice de satisfação do usuário com um software de gestão contribui para melhorar a qualidade da tomada de decisão das organizações, avaliando como variáveis de qualidade do sistema impactam na satisfação do usuário (CHEN, 2010), propostas de modelos capazes de medir a satisfação com o uso do sistema (PIKE et al, 2011; STRAKA, 2010; JIANG; SHANG; LIU, 2010; CHEN, 2008), e também diagnósticos de satisfação considerando construtos como a heterogeneidade de consumo (RAMASUBU; MITHAS; KRISHNAM, 2008), aprendizagem organizacional (ONG; LAI, 2007) e, ainda pesquisas que utilizam dados dos sistemas para medir a satisfação (ONG; DAY; HSU, 2009).

Desta forma tão importante quanto à qualidade técnica do software, ou sistema de informação, é a avaliação que seus usuários fazem do mesmo, tanto para dar retorno a empresa sobre a compensação de seu investimento, bem como para gerar informações que possam colaborar para o desenvolvimento da organização.

Tendo como base a importância sobre a satisfação dos usuários para o melhor aproveitamento do sistema, esta pesquisa se propõe em seu objetivo medir o nível de satisfação dos usuários do sistema de gestão hospitalar - SmartHealth, na organização objeto do estudo, buscando como objetivos específicos; (i) Identificar as dimensões que melhor se relacionam ao sistema SmartHealth e (ii) Identificar as dimensões que compõem a percepção de satisfação dos usuários no uso do sistema

O artigo está dividido em 6 tópicos, incluindo este tópico introdutório. Em seguida no tópico 2, discute-se sobre a avaliação dos sistemas de informação e, em seguida no tópico 3 são descritos diversos modelos de satisfação do usuário de sistemas de informação. No tópico 4 é descrito a caracterização do estudo, o plano de coleta e análise dos dados. No tópico 5 são apresentados e discutidos os resultados da pesquisa. Por fim, o último tópico é referente às considerações finais.

\section{AVALIAÇÃO DE SI SOB A PERSPECTIVA DO USUÁRIO}


Diante da realidade do mercado atual, diversas organizações tem investido recursos significantes na implementação de softwares de apoio a decisão para modernizar e integrar a infraestrutura do processo de negócio e medir a satisfação do usuário de softwares (RAMASUBU; MITHAS; KRISHNAN, 2008; SILVEIRA; ZWICKER, 2004).

De acordo com Abu-Musa (2002), a avaliação dos sistemas de informação (SI) tem como principais finalidades: verificar se o SI está de acordo com o que foi solicitado; proporcionar retroalimentação para o desenvolvimento de usuários; justificar a aquisição, a continuidade ou o término de um projeto de SI e ainda identificar mudanças necessárias. Assim, na fase de avaliação do SI, é possível identificar problemas ou oportunidades em potencial e verificar se o SI está ou não contribuindo adequadamente para que os objetivos e as metas da empresa sejam atingidos. O trabalho de Pike et al (2011) relaciona dentre outros construtos o uso da informação com a ação de decisão quanto ao uso de um software, transparecendo a influência que a utilização de um software pode desempenhar frente a tomada de decisão de uma organização.

Em termos da avaliação do SI, uma das formas de medir a qualidade da informação é através da mensuração da satisfação do usuário com o SI. As medidas de satisfação são as mais utilizadas em pesquisas de avaliação do sucesso de sistemas de informação. (IGBARIA; NACHMAN, 1990; MELONE, 1990; CANHETTE, 2004).

De acordo com Canhette (2004) quando existe uma satisfação positiva dos usuários com os sistemas de informação que utilizam, há como indicação de que estes contribuem na execução de suas atividades e, em consequência, têm um impacto positivo em seu desempenho e no desempenho da organização.

Dentre outros benefícios, a avaliação do sucesso de um sistema de informação e a satisfação do usuário são pontos importantes para a uma melhor tomada de decisão no que diz respeito a sistemas de gestão que utilizam plataformas virtuais e tecnologias da internet (ONG; DAY; HSU, 2009). Seguindo o mesmo raciocínio, Ong e Lai (2007) afirmam que medir a satisfação dos usuários torna os gestores capazes de reordenar o pensamento e resolver intrincados problemas no ambiente de trabalho. Convencionalmente, um sistema recomendado é considerado um sucesso se os clientes/usuários recomendam o uso do sistema (JIANG; SHANG; LIU, 2010).

Não se fazendo distinção entre dois tipos de usuários. Segundo Delone e Mclean (1992 apud Ely, 2010) é possível elencar três motivos que justificam a medida de satisfação do usuário como uma das mais utilizadas para avaliar o sucesso de um sistema de informação:

I. A satisfação tem um alto valor de face: é difícil negar o sucesso de um sistema com o qual os usuários dizem estar satisfeitos;

II. O desenvolvimento de instrumentos, como ferramentas confiáveis para medir a satisfação, como os de Bailey e Person (1983 apud Ely, 2010) e Wu e Wang (2007 apud Ely, 2010), proporcionou parâmetros importantes para futuros estudos sobre satisfação.

III. Muitas vezes acaba-se utilizando a satisfação do usuário porque as demais medidas ou são conceitualmente fracas, ou são empiricamente difíceis de serem obtidas.

Nesta pesquisa o usuário é definido sob as perspectivas dos conceitos apresentados podendo ser definido como um indivíduo que usa um recurso disponibilizado, onde a informação é imprescindível para o desenvolvimento do seu trabalho, sendo o mesmo, ator gerador das informações processadas no SI, e, portanto importante para o sucesso dos sistemas de informação (RAMALHO et al, 2009).

\section{MODELOS DE AVALIAÇÃO DE SI SOB A PERSPECTIVA DO USUÁRIO}

Diversos modelos podem ser utilizados quando se avalia um SI. De acordo com Zwass (1992), é fundamental determinar qual informação é necessária e a qualidade dessa 
informação, pois quando se implementa um sistema de informação espera-se que este forneça informações com a qualidade esperada.

Na pesquisa de Sternad, Gradisar e Bobek (2011) são investigados fatores que impactam na satisfação do usuário, que por sua vez determinam a eficiência e efetividade de um sistema de gestão integrado, através da aceitação e do uso da tecnologia empregada na organização. Para tanto, foi utilizado o Modelo de Aceitação de Tecnologia (TAM) desenvolvido por Davis (1989). Já no estudo de Saatçioglu (2009), a proposta de avaliação do sistema sob a percepção do usuário foi feita através da investigação de 4 construtos: efeitos, benefícios, barreiras e riscos. Os resultados da pesquisa geraram subsídios no processo de tomada de decisão, tais subsídios são necessários devidos aos altos investimentos que um sistema ERP requer.

Dentre os modelos adotados para as avaliações dos sistemas, em alguns casos busca-se um modelo adaptável para determinadas organizações que apresentam certas particularidades no sistema utilizado. No estudo de Longinidis e Gotzamani (2009) foi realizado um estudo de satisfação dos usuários de um ERP sob a percepção dos usuários de uma organização de distribuição de energia. Nos casos de criação de novos modelos ou remodelações de modelos pré-existentes, são propostos com vista a atualizar as lacunas da literatura da área ao longo do tempo. Na pesquisa de Neto (2003) foi proposto o desenvolvimento de um instrumento capaz de medir a satisfação dos usuários de um ERP para validar empiricamente um modelo capaz de se tornar uma ferramenta de diagnóstico na implementação de sistemas de informação, aumentando as chances de sucesso do sistema.

Além de auxiliar no processo decisório e determinar o sucesso de um sistema, os modelos presentes na literatura focados na experiência do usuário apresentados até aqui também buscam maximizar a integração no ambiente de trabalho e melhorar a relação da organização com os clientes (DOLL; TORKZADEH, 1999; WU; WANG, 2007; WU et al, 2002; ELY, 2010).

\section{ESTRATÉGIA METODOLÓGICA E PERFIL DA AMOSTRA}

O presente artigo é resultado de uma pesquisa exploratório-descritiva, de acordo com características definidas por Vergara (2006) e Gil (1991). Buscando atender ao objetivo geral da pesquisa que é identificar o nível de satisfação dos usuários do sistema de gestão hospitalar - SmartHealth, na organização objeto do estudo, o presente artigo pretende medir, descrever e possibilitar exploração dos dados coletados e analisados de acordo com Bryman (1989), Malhotra (2001) e Boente (2004).

Este estudo foi aplicado num hospital privado localizado na cidade de Natal-RN, caracterizando esta pesquisa também como um estudo de caso, tendo em vista que o campo da pesquisa está restrito a uma organização. O nome da empresa foi preservado por questões éticas sendo identificada por Hospital Viver Bem.

Da totalidade de funcionários atuantes no Hospital Viver Bem (HVB), que conta atualmente com 684 indivíduos, foram considerados como universo desta pesquisa, apenas àqueles que possuem acesso ao sistema SmartHealth. A população total da pesquisa contou com 290 colaboradores da organização estudada. É reconhecido que mais de $90 \%$ da população de colaboradores utiliza o sistema regularmente. Dessa forma, para o cálculo da amostra, foi levada em consideração o percentual mínimo de $90 \%$ e um nível de confiança de $95 \%$, com um erro amostral de 0,05 . O procedimento de cálculo é referente a amostragens aleatórias simples com variáveis categóricas, como define Santos (2012), sendo a mais adequada para o presente estudo. O cálculo é realizado de acordo com a fórmula descrita abaixo: 


$$
n=\frac{N \cdot Z^{2} \cdot p \cdot(1-p)}{Z^{2} \cdot p \cdot(1-p)+e^{2} \cdot(N-1)}
$$

Onde $\mathrm{n}$ - amostra calculada; N - população; $\mathrm{Z}$ - variável normal padronizada associada ao nível de confiança; $\mathrm{p}$ - verdadeira probabilidade do evento; e - erro amostral. Após realizado o cálculo, a amostra requerida para o estudo foi de 94 casos ou observações. Foram registrados 113 casos para o estudo, sendo maior do que a amostra requerida e coerente com o que é indicado por Hair et al (2005).

A coleta de dados ocorreu no início de janeiro/2012, através da aplicação dos questionários pelos pesquisadores, com a contribuição de divulgação da pesquisa pela gestora de TI do HVB. Foi realizado um pré-teste com 50 usuários do sistema na organização no intuito de validar a utilização das variáveis do instrumento e verificar a adequabilidade da redação aos termos conhecidos pelos respondente. Após adequação do questionário, foram aplicados 133 questionários com todos os usuários da população da pesquisa levando em conta a disponibilidade dos participantes no momento de aplicação do instrumento de pesquisa. Destes, foram descartados 20 questionários que não foram $100 \%$ respondidos e a amostra final da pesquisa ficou representada com 113 questionários.

Foi empregado nesta pesquisa um questionário estruturado com dois modelos conforme dois grupos de usuários, cuja versão original foi elaborada e adaptada por Ely (2010). O modelo final de Ely foi elaborado a partir da combinação do modelo para medir a satisfação do usuário final de um sistema, adaptado a partir do modelo desenvolvido e validado por Wu et al (2002) combinado com o modelo de Wu e Wang (2007) e Torkzadeh e Doll (1999) para medir a satisfação dos usuários-chave de um sistema de gestão empresarial ERP.

O perfil da amostra de 113 usuários do sistema SmartHealth no Natal Hospital Center, se apresenta em sua grande maioria por indivíduos do sexo masculino $(66,4 \%)$, idade entre 21 e 40 anos $(85,8 \%)$ e com grau de escolaridade com graduação completa ou incompleta $(52,2 \%)$, apresentando ainda $28,3 \%$ dos respondentes com ensino médio. Quanto ao vínculo com a organização a maioria $(93,81 \%)$ são funcionários, e a minoria terceirizados, com tempo de serviço concentrado em menos de 1 ano a 3 anos (66,3\%). A maioria dos respondentes ocupa o nível operacional (78,8\%), sendo a maior concentração no setor de Apoio Administrativo (76,1\%). Na Tabela 01 abaixo é possível visualizar os dados do perfil da amostra com mais detalhes.

Tabela 01: Perfil da amostra

\begin{tabular}{cccc}
\hline PERFIL DOS RESPONDENTES & Qtd & $\%$ \\
\hline Gênero & & \\
\hline Masculino & 35 & 66,40 \\
Feminino & & 33,60 \\
\hline Idade & 3 & 2,70 \\
\hline Menor de 20 anos & 50 & 44,20 \\
21 a 30 anos & 47 & 41,60 \\
31 a 40 anos & 11 & 9,70 \\
41 a 50 anos & 2 & 1,80 \\
\hline Acima de 50 anos & & \\
\hline Escolaridade & & 28,30
\end{tabular}




\begin{tabular}{ccc} 
Graduação Incompleta & 20 & 17,70 \\
Graduação Completa & 39 & 34,50 \\
Curso Técnico & 7 & 6,20 \\
Pós Graduação Incompleta & 7 & 6,20 \\
Pós Graduação Completa & 8 & 7,10 \\
\hline Tempo que trabalha no NHC & 24 & 21,20 \\
\hline Menos de 1 ano & 51 & 45,10 \\
Entre 1 e 3 anos & 19 & 16,80 \\
Entre 4 e 7 anos & 17 & 15,00 \\
Entre 8 e 11 anos & 2 & 1,80 \\
Mais de 11 anos & \multicolumn{3}{|c}{} \\
\hline Posição ocupada no NHC & 22 & 19,50 \\
Nível de Chefia & 89 & 78,80 \\
Nível Operacional & 2 & 1,80 \\
\hline Estagiário/Aprendiz & \multicolumn{3}{c}{}
\end{tabular}

Fonte: Dados da Pesquisa, 2012.

\section{ANÁLISE DOS RESULTADOS}

\subsection{DIMENSÕES DA SATISFAÇÃO NO USO DO SISTEMA DE INFORMAÇÃO}

Para atender a um dos objetivos específicos deste estudo que é identificar as dimensões que compõem um conjunto de características do sistema que leva a percepção de satisfação dos usuários no uso do sistema, foi utilizada a técnica estatística de Análise Fatorial Exploratória (AFE), com cinco dimensões: 'acuracidade'; 'confiabilidade'; 'tempo de resposta'; 'completeza'; 'disponibilidade da informação'. Sendo cada uma das variáveis medidas por quatro quesitos, totalizando 32 variáveis. O conjunto de variáveis foi adaptado dos estudos de Ely (2010) e Wu e Wang (2007). Dessa forma, a análise fatorial teve como objetivo principal a redução dos dados de forma a encontrar dimensões latentes, mas não observáveis diretamente (HAIR, et al., 2005) facilitando a análise dos fatores realmente influenciáveis na satisfação dos usuários desta pesquisa de campo.

Para averiguar se a análise fatorial era apropriada, inicialmente foi realizada a inspeção da matriz de correlações, a fim de verificar a conformidade com os pressupostos: Correlação; Anti-Imagem; Comunalidade; Carga Fatorial e Cross Load, para cada uma das variáveis do modelo, também foram realizados os testes Keiser-Meyer-Olkin (KMO) para checar a adequação da amostra e o Teste de Esfericidade de Bartlett, que avalia a presença de correlações entre as variáveis, medindo a significância do modelo, conforme sugerido por Hair et al (2005).

Nos pressupostos de Comunalidade, Correlação e Anti-Imagem todas as variáveis apresentaram bons índices. Ao verificar o pressuposto de Carga Fatorial que serve como parâmetro para verificar o pressuposto de Cross Load, não passaram os quesitos da dimensão 'completeza', sendo esta retirada do modelo, e alguns quesitos da dimensão 'disponibilidade', como é demostrado na Tabela 02. Dessa forma, das 32 variáveis contou-se no modelo final para aplicação da análise fatorial e formação das dimensões com 26 variáveis. 
Tabela 02: Variáveis excluídas para aplicação da AFE

\begin{tabular}{|c|c|c|c|c|c|}
\hline \multicolumn{6}{|c|}{ Variáveis Excluídas sob o Critério de Exclusão Cross Load } \\
\hline Completeza & $\begin{array}{c}\text { Índice } \\
D 1^{*}\end{array}$ & $\begin{array}{c}\text { Índice } \\
D 2^{*}\end{array}$ & Disponibilidade & $\begin{array}{c}\text { Índice } \\
D 1^{*}\end{array}$ & $\begin{array}{c}\text { Índice } \\
D 2^{*}\end{array}$ \\
\hline Quesito Consistente & 0,580 & 0,552 & Quesito Em Teтpo & 0,682 & 0,418 \\
\hline Quesito Completo & 0,562 & 0,578 & Quesito Razoável & 0,706 & 0,419 \\
\hline Quesito Adequado & 0,586 & 0,516 & & & \\
\hline Quesito Suficiente & 0,629 & 0,421 & & & \\
\hline
\end{tabular}

*Onde $D 1$ e $D 2$, correspondem às dimensões que poderiam conter estas variáveis. Cross load: não permite que uma mesma variável esteja enquadrada em mais de uma dimensão, apresentando carga fatorial a partir de 0,4 , nas dimensões formadas.

Fonte: Dados da Pesquisa, 2012.

No teste KMO foi obtido o valor de 0,856 , o que representa que a amostra é adequada para a análise fatorial. Conforme Hair et al (2005), a medida pode ser interpretada como adequada quando atinge índices acima de 0,7, estando o KMO desta amostra acima do índice de 0,80, considerado como admirável. No teste de Esfericidade de Bartlett, o nível de significância foi de 0,00 , o que indica que existem correlações significativas entre as variáveis. Pois como explica Hair et al (2005) quanto mais próximo de zero melhor.

Para a análise fatorial, foi utilizada uma análise fatorial exploratória (AFE) convergente, dentro de cada dimensão. O método de determinação de fatores foi o de Análise de Componentes Principais, por utilizar a variância total dos itens e representar, em um mínimo de fatores, o máximo de variância possível. Em conjunto foi aplicado o método Varimax de rotação ortogonal, para melhorar a interpretabilidade dos fatores e mantê-los independentes. A análise utilizada foi a matriz de correlação com o máximo de 25 interações por convergência. As dimensões que obtiveram o Eigenvalue acima de 1, foram consideradas para este estudo, sendo um total de 6 (seis) dimensões consideradas, que explicam o objeto de estudo em $87,78 \%$, sendo este valor o total da variância explicada pelas dimensões.

O Alpha de Cronbach $(\alpha)$, sendo uma medida de confiabilidade, foi calculado para todas as dimensões formadas uma vez que todas se mostraram adequadas, atingindo índices acima de 0,9 , já que ela varia de 0 a 1 , sendo os valores de 0,60 a 0,70 considerados o limite inferior de aceitabilidade (HAIR et al, 2005). Os índices de Alpha de Cronbach de cada dimensão encontrada, bem como as cargas fatoriais das variáveis manifestas são apresentados na Tabela 03.

Tabela 03: Dimensões da satisfação no uso do sistema Smart

\begin{tabular}{lllll}
\hline Dimensões e variáveis & $\begin{array}{l}\text { Carga } \\
\text { Fatorial }\end{array}$ & Eingenvalue & Variância (\%) & (a) \\
\hline 1- Confiança & & 10,70 & 25,065 & 0,962 \\
\hline 'Acuracidade' - Quesito Exatas & 0,884 & & \\
'Acuracidade' - Quesito Confiabilidade & 0,861 & & \\
'Acuracidade' - Quesito Consistência & 0,901 & & \\
'Acuracidade' - Quesito Suficiência & 0,827 & & \\
'Confiabilidade'-Quesito Consistência & 0,799 & & \\
'Confiabilidade'-Quesito Alta & 0,832 & & \\
\hline
\end{tabular}


Revista de Administração Hospitalar, v.11, n.1, pp. 28-41, janeiro/março, 2014/ Sheila de Lima Cunha Dantas e Fernando Antônio de Melo Pereira

\begin{tabular}{|c|c|c|c|c|}
\hline $\begin{array}{l}\text { 'Confiabilidade' - Quesito Superioridade } \\
\text { 'Confiabilidade' - Quesito Suficiência }\end{array}$ & $\begin{array}{l}0,861 \\
0,857 \\
\end{array}$ & & & \\
\hline 2-Treinamento & & 1,611 & 15,107 & 0,984 \\
\hline 'Treinamento' - Quesito Completo & 0,941 & & & \\
\hline 'Treinamento' - Quesito Alto & 0,935 & & & \\
\hline 'Treinamento'- Quesito Suficiente & 0,926 & & & \\
\hline 'Treinamento'-Quesito Superior & 0,939 & & & \\
\hline 3-Tempo de Resposta & & 1,527 & 14,555 & 0,963 \\
\hline 'Tempo de Resposta'-Quesito Aceitável & 0,806 & & & \\
\hline 'Tempo de Resposta' - Quesito Consistente & 0,903 & & & \\
\hline 'Tempo de Resposta' - Quesito Rapidez & 0,911 & & & \\
\hline 'Tempo de Resposta'-Quesito Qualidade & 0,920 & & & \\
\hline 4-Envolvimento & & 1,282 & 13,707 & 0,943 \\
\hline 'Envolvimento' - Quesito Alto & 0,852 & & & \\
\hline 'Envolvimento'-Quesito Forte & 0,844 & & & \\
\hline 'Envolvimento'-Quesito Positivo & 0,900 & & & \\
\hline 'Envolvimento' - Quesito Envolvido & 0,888 & & & \\
\hline 5-Conhecimento & & 1,103 & 12,868 & 0,942 \\
\hline 'Conhecimento'-Quesito Completo & 0,875 & & & \\
\hline 'Conhecimento' - Quesito Alto & 0,879 & & & \\
\hline 'Conhecimento'-Quesito Suficiente & 0,852 & & & \\
\hline 'Conhecimento' - Quesito Didático & 0,731 & & & \\
\hline 6-Disponibilidade da Informação & & 1,103 & 6,485 & 0,926 \\
\hline $\begin{array}{l}\text { 'Disponibilidade da Info.' - Quesito Disponivel } \\
\text { 'Disponibilidade da Info.' - Quesito Consistente }\end{array}$ & $\begin{array}{l}0,790 \\
0,813\end{array}$ & & & \\
\hline Variância total explicada & & & 87,787 & \\
\hline
\end{tabular}

Método de extração: Análise de Componentes Principais Método de Rotação: Varimax with Kaiser Normalization KMO (Kaiser-Meyer-Olkin): 0,856. Bartlett's Test of Sphericity: 5142,098 (Sig, 0,000).

Fonte: Dados da Pesquisa, 2012.

\subsection{AVALIAÇÃO DA SATISFAÇÃO GERAL COM O SMART}

Um dos principais tópicos do estudo é em relação à satisfação geral dos usuários com o sistema Smart. A satisfação é um construto presente em diversos estudos que abordam a percepção de usuários em relação a produtos e serviços. Nesse sentido, a avaliação da satisfação geral do usuário com o sistema Smart é apresentada como variável dependente, na análise de regressão, tendo como variáveis independentes as dimensões de qualidade do sistema identificadas através da AFE. Tais variáveis foram submetidas a uma análise de regressão múltipla, com vista a identificar quais dimensões apresentam mais influência na determinação da satisfação geral e medir a força de tais influências.

Para regressão múltipla, foi utilizado o método Stepwise, por ser considerado o mais adequado uma vez que se evita a multicolinearidade entre as dimensões do modelo. O método Stepwise utiliza como parâmetro de inclusão de fatores os valores de probabilidade iguais ou menores que 0,05 e para a exclusão os valores iguais ou superiores a 1 .

Através da análise realizada chegou-se a um modelo constituído por três variáveis independentes, após serem submetidas a diversos testes de violação de pressupostos. Para a configuração do modelo final encontrado pela análise foram considerados cinco pressupostos a serem testados. Se os pressupostos não forem seguidos, portanto, as estimativas podem ser inconsistentes e enviesadas, o que implicará em maior erro padrão, ou maior dispersão em torno da reta e prejudicará a análise de regressão (CORRAR; PAULO; DIAS FILHO, 2009). 
Os pressupostos requeridos foram: normalidade dos resíduos, homoscedasticidade dos resíduos, linearidade dos coeficientes, ausência de autocorrelação serial nos resíduos e multicolinearidade, seguindo as recomendações de Corrar, Paulo e Dias Filho (2009). Os resultados dos pressupostos são apresentados na Tabela 04.

Tabela 04: Avaliação de pressupostos do modelo de regressão múltipla.

\begin{tabular}{|c|c|c|c|}
\hline Teste & Índice & Valores de referência & Diagnóstico \\
\hline Kolmogorov-Smirnov & $\begin{array}{c}K . S=0,507 \\
\text { Asymp. Sig. }(2 \text {-tailed })= \\
0,959\end{array}$ & $\begin{array}{c}\text { Se Sig. > 0,05 (erro } \\
\text { amostral) }\end{array}$ & $\begin{array}{c}\text { A distribuição é } \\
\text { normal }\end{array}$ \\
\hline Pesarán-Pesarán & $F=0,195 ;$ Sig. 0,660 & $\begin{array}{c}\text { Se Sig. > 0,05 (erro } \\
\text { amostral) }\end{array}$ & $\begin{array}{c}\text { Dispersão homogênea } \\
\text { (homoscedasticos) }\end{array}$ \\
\hline Tolerance & $\begin{array}{l}\text { Fator } 2=1 ; \text { Fator } 5=1 ; \\
\text { Fator } 6=1\end{array}$ & $\begin{array}{l}\text { De } 1 \text { até } 10-\text { com } \\
\text { multicolinearidade } \\
\text { aceitável. }\end{array}$ & \multirow{2}{*}{$\begin{array}{l}\text { Multicolinearidade } \\
\text { aceitável }\end{array}$} \\
\hline$V I F$ & $\begin{array}{l}\text { Fator } 2=1 ; \text { Fator } 5=1 \\
\text { Fator } 6=1\end{array}$ & $\begin{array}{c}\text { De } 1 \text { até } 0,10-\text { com } \\
\text { multicolinearidade } \\
\text { aceitável }\end{array}$ & \\
\hline Linearidade & $\begin{array}{c}\text { Avaliação gráfica } \\
\text { (diagramas de dispersão) }\end{array}$ & - & $\begin{array}{c}\text { As variáveis são } \\
\text { lineares }\end{array}$ \\
\hline Durbin-Watson & 2,046 & Valores próximos de 2 & $\begin{array}{c}\text { Ausência de } \\
\text { autocorrelação serial }\end{array}$ \\
\hline
\end{tabular}

Fonte: Dados da pesquisa, 2012.

De acordo com a Tabela 04, todos os testes apresentaram índices adequados, demonstrando que o procedimento estatístico foi aplicado de forma apropriada mediante o cumprimento do conjunto de pressupostos. A escolha dos testes aplicados para atestar o cumprimento dos pressupostos se deu mediante orientações de Hair et al. (2005) e de Corrar, Paulo e Dias Filho (2009).

O modelo final de regressão do estudo é constituído pelas dimensões Confiança (com as variáveis 'acuracidade' e 'confiabilidade'), Tempo de resposta e Envolvimento. Juntas, as dimensões possuem poder explicativo de 31,4\%, considerado razoável. O resultado do teste F-ANOVA obteve significância zero (Sig. 0,000), permitindo afirmar que a hipótese de que $\mathrm{R}^{2}$ é igual à zero pode ser rejeitada, logo, é possível afirmar que as variáveis estatísticas possuem influência sobre a variável dependente e o modelo no todo, pode ser considerado significativo. Além disso, o teste $\mathrm{T}$ com significância menor que 0,05 para todas as dimensões corroboram a adequabilidade e significância estatística do modelo encontrado (CORRAR; PAULO; DIAS FILHO, 2009), que pode ser observado na Tabela 05.

Tabela 05: Fatores influenciadores da Satisfação Geral

\begin{tabular}{lccccc}
\hline $\begin{array}{c}\text { Variáveis } \\
\text { independentes }\end{array}$ & $\begin{array}{c}\boldsymbol{\beta} \text { não } \\
\text { padronizado }\end{array}$ & $\begin{array}{c}\text { Erro } \\
\text { padrão }\end{array}$ & $\boldsymbol{\beta}$ Padronizado & Teste t & Sig. \\
\hline (constante) & 5,422 & 0,094 & - & 57,657 & 0,000 \\
\hline Confiança & 0,508 & 0,137 & 0,425 & 5,355 & 0,000 \\
Tempo de resposta & 0,360 & 0,137 & 0,301 & 3,794 & 0,000
\end{tabular}




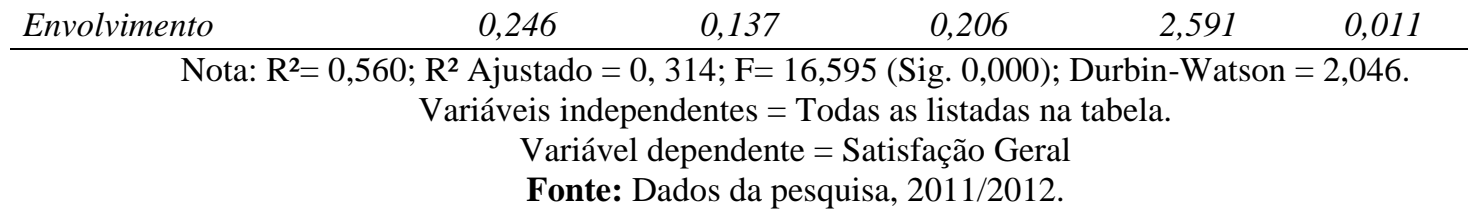

De acordo com os resultados apresentados na Tabela 05 a dimensão que exerce maior influência na explicação da satisfação geral com o sistema é a Confiança $(\beta=0,425)$. Os resultados também mostram que os usuários denotam importância ao Tempo de resposta do sistema $(\beta=0,301)$ e o Envolvimento $(\beta=0,206)$, sendo fatores decisivos para determinar a satisfação do usuário com o sistema.

A dimensão de Confiança que traduz a acuracidade e confiabilidade das informações encontradas no sistema, apontada pelo modelo como a principal dimensão para medir a satisfação do usuário, pode ser explicada conforme visto na revisão da literatura desta pesquisa, que a qualidade da informação fornecida pelo sistema é fundamental para medir a qualidade esperada por um sistema de informação (ZWASS, 1992), onde exatidão, medida pela acuracidade, e a consistência das informações fornecidas, medida pela confiabilidade representam significância para o nível de satisfação dos usuários quanto ao sistema.

O Tempo de resposta, indicado como outra dimensão importante na satisfação do usuário com o sistema, pode ser explicado pelo fato da organização objeto do estudo de caso se tratar de uma unidade hospitalar, onde o tempo é muito valorizado e muitas vezes crucial para o adequado atendimento ao paciente, dessa forma, contar com um sistema que seja rápido e eficiente no tempo de resposta torna-se um fator relevante para os respondentes que atuam na unidade hospitalar.

A dimensão Envolvimento, apontada como outro fator influenciador na satisfação do usuário na pesquisa de campo revela que o usuário tem consciência de que sua participação e interesse são fundamentais para o bom desempenho do sistema de informação, sendo um fator positivo para a organização, uma vez que são os usuários que alimentam as informações no sistema, quanto mais envolvidos, maior será o índice de acuracidade e confiabilidade das informações oferecidas pelo Smart, e maior será o nível de satisfação do próprio usuário.

Para a validação dos resultados, foi adotado o que Corrar, Paulo e Dias Filho (2009) recomendam. Os autores sugerem que a amostra coletada seja repartida em chamadas sub amostras de forma aleatória, sendo processadas análises de regressão múltiplas para cada uma delas independentemente para posterior comparação com o modelo de regressão completo. Sendo assim, os resultados foram validados a partir dos resultados de duas amostras, cada uma contendo $50 \%$ dos casos observados. Os principais resultados comparativos estão sumarizados na Tabela 06.

Tabela 06: Validação dos resultados do modelo de regressão linear múltipla

\begin{tabular}{ccccc}
\hline Estatísticas & Amostra geral & Amostra 50\% & Amostra 50\% \\
\hline R2 ajustado & 0,314 & 0,295 & 0,274 \\
Estatística $F$ & 16,595 & 13,538 & 19,499 \\
Durbin Watson & 2,046 & 2,103 & 2,059 \\
VIF (as três variáveis) & 1 & 1 & 1 \\
\hline
\end{tabular}

Fonte: Dados da pesquisa, 2012.

Os resultados comparativos mostrados na Tabela 06 demonstram um comportamento razoavelmente homogêneo, não se detectando qualquer parâmetro que destoe de maneira acentuada. Sendo assim, procedidas às adaptações necessárias para adequação do modelo, as variações explicadas e os testes realizados se apresentam validos, constituindo o modelo final encontrado pela pesquisa, representando pela Figura 01. 


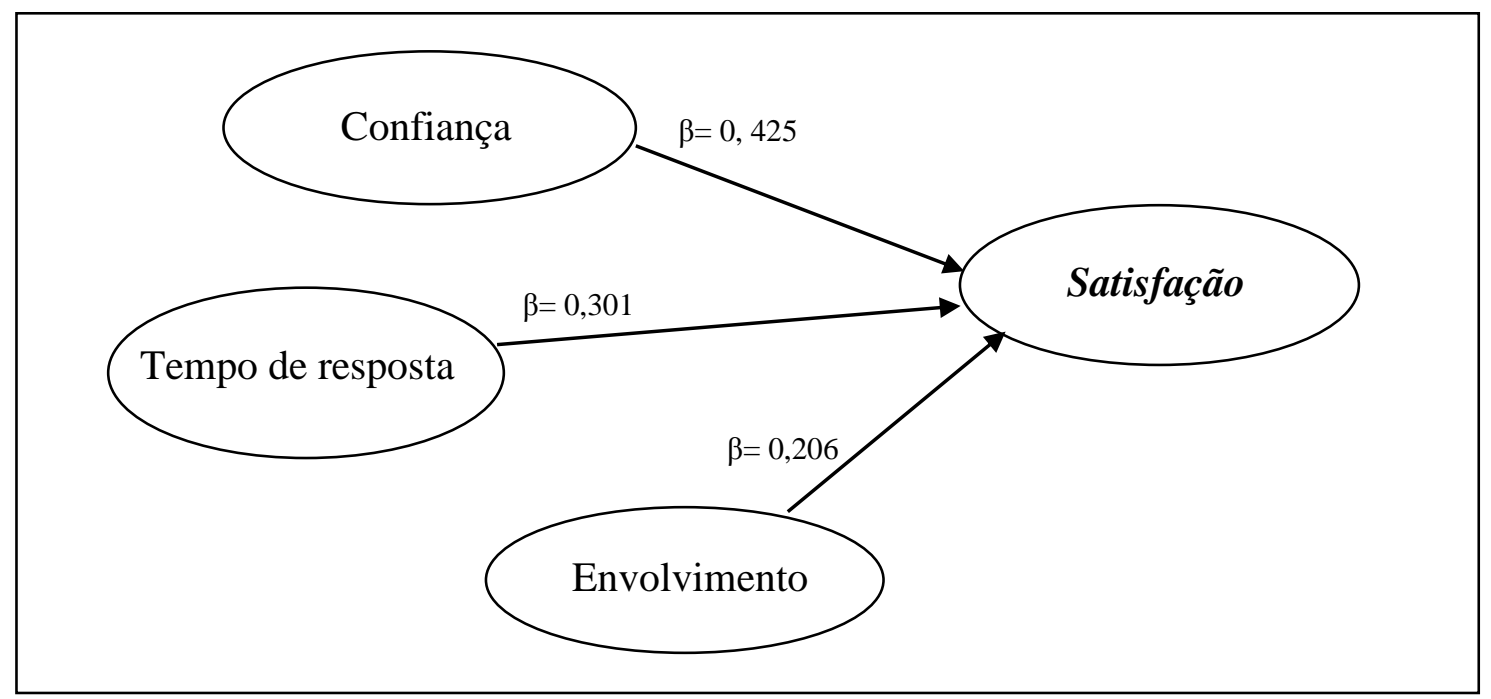

Figura 01: Modelo encontrado pelo estudo.

Fonte: Dados da pesquisa, 2012.

\section{CONSIDERAÇÕES FINAIS}

A pesquisa, realizada no Hospital Viver Bem (HVB), que teve seu nome preservado por questões éticas, localizado na cidade de Natal/RN, buscou como seu objetivo geral identificar o nível de satisfação dos usuários do sistema de gestão hospitalar - SmartHealth, usando a satisfação do usuário, conforme recomendado pela literatura, como medida para verificar o desempenho do sistema Smart no HVB. Em geral, os usuários do sistema Smart no Hospital Viver Bem, encontram-se satisfeitos com o sistema. Foi possível constatar que a frequência e o tempo de uso do sistema também se mostraram relevantes para medição da satisfação, tendo em vista que quanto mais se utiliza o sistema, mais fácil será avaliá-lo.

Pode-se afirmar que o objetivo proposto foi atingido, permitindo apontar as dimensões ('confiança', 'tempo de resposta', 'envolvimento') como influenciadores na satisfação de seus usuários, sendo considerada esta a principal contribuição da pesquisa para a organização estudada, bem como podendo servir como instrumento para aplicação de pesquisas futuras em outras unidades hospitalares.

No entanto, a pesquisa apresenta como limitações o fato de ter sido aplicada em apenas uma organização hospitalar e não incorporar ao instrumento de pesquisa outros modelos capazes de mensurar a satisfação dos usuários de um sistema. O modelo encontrado pela pesquisa pode não apresentar os mesmo resultados em outras aplicações, cabendo a estudos futuros investigar linguagem, cultura, regionalidade e influências políticas em determinadas organizações. Portanto, o modelo encontrado é adaptável e pode ser replicado em outras organizações.

É sugerido ainda que modelos teóricos que avaliem outros aspectos inerentes à utilização de sistemas de informação em ambientes hospitalares sejam aplicados para validar empiricamente diversos instrumentos que possibilitem gerar informações capazes de influenciar positivamente o processo de tomada de decisão dos gestores aproveitando ao máximo a capacidade de sistemas de gestão hospitalares. 
Revista de Administração Hospitalar, v.11, n.1, pp. 28-41, janeiro/março, 2014/ Sheila de Lima Cunha Dantas e Fernando Antônio de Melo Pereira

\section{REFERÊNCIAS}

ABU-MUSA, Ahmad A. Security of computerized accounting information systems: an integrated evaluation approach. The Journal of American Academy of Business. Cambridge, 2002.

BOENTE, Alfredo; BRAGA, Glaucia. Metodologia científica contemporânea para universitários e pesquisadores. 2. ed. Rio de Janeiro: Brasport, 2004.

BRYMAN, A. Research methods and organization studies. London: Routledge, 1989.

CANHETE, Cláudio Cesar. Análise das menções à qualidade das informações em teses e dissertações que relatam impacto do uso de sistemas ERP. Dissertação de Mestrado. Universidade de São Paulo, 2004.

CHEN, Ling Hsiu. Job satisfaction among information system (IS) personnel. Computers in Human Behavior. v. 24, n. 1, p. 105-118, 2008.

CHEN, Ching Wen. Impact of quality antecedents on taxpayer satisfaction with online tax filing systems: an empirical study. Information \& Management. v. 47, n. 6, p. 308-315, 2010.

CORRAR, Luiz; PAULO, Edilson; DIAS FILHO, José Maria. Análise Multivariada: Para os cursos de administração, ciências contábeis e economia. São Paulo: Atlas, 2009.

DIAS, D. de S. Eficácia de sistemas de informação, participação do usuário e mudança organizacional. Anais, ANPAD, v.2, Salvador, 1993.

ELY, Patrícia Bins. Medindo a satisfação dos usuários finais e dos usuários chave de um sistema de gestão empresarial, na empresa Springer Carrier. Dissertação de Mestrado. Universidade Federal do Rio Grande do Sul - UFRGS, 2010.

GIL, Antônio Carlos. Como elaborar projetos de pesquisa. São Paulo: Atlas, 1991.

HAIR, J.F, Jr.; BLACK, W.C.; BABIN, B.J.; ANDERSON, R.E.; TATHAN, R.L. Análise Multivariada de Dados. 6 ed. Porto Alegre: Bookman, 2005.

JIANG, Yuanchun; SHANG, Jennifer; LIU, Yezheng. Maximizing customer satisfaction through an online recommendation system: a novel associative classification model. Decision Support Systems. v. 48, n. 3, p. 470-479, 2010.

LONGINIDIS, Pantelis; GOTZAMANI, Katerina. ERP user satisfaction issues: insights from a Greek industrial giant. Industrial Management \& Data Systems. v. 109, n. 5, p. 628-645, 2009.

MALHOTRA, Naresh K. Pesquisa de Marketing. Porto Alegre: Bookman, 2001.

NETO, José Dutra de Oliveira. Desenvolvimento de um instrumento para mensurar a satisfação do usuário de sistemas. Thirteenth Asian-Pacific Conference on International Accounting Issues. Rio de Janeiro/RJ, Brasil, 2001. 
ONG, Chorng Shyong; DAY, Min Yuh; HSU, Wen Lian. The measurement of user satisfaction with question answering systems. Information \& Management. v. 46, n. 7, p. 397403, 2009.

ONG, Chorng Shyong; LAI, Jung Yu. Measuring user satisfaction with knowledge management systems: scale development, purification, and initial test. Computers in Human Behavior. v. 23, n. 3, p. 1329-1346, 2007.

PIKE, Richard H.; TAYLES, Mike E. NAHA, Nur; MANSOR, Abu. Activity based costing user satisfaction and type of system: a research note. The British Accounting Review. v. 43, n. 1, p. 65-72, 2011.

PRESSMAN, R. S. Engenharia de software. Tradução José Carlos Barbosa dos Santos 4. ed. São Paulo: Makron Books, 1995.

RAMALHO, Francisca Arruda; SILVA, Alan C. P.; COSTA, Luciana F. (Re)visitando os estudos de usuário: entre "tradição" e o "alternativo". Datagamazero, v.10, n. 04, 2009.

RAMASUBU, Narayan; MITHAS, Sunil; KRISHNAN, M. S. High tech, high touch: the effect of employee skills and customer heterogeneity on customer satisfaction with enterprise system support services. Decision Support Systems. v. 44, n. 2, p. 509-523, 2008.

SAATÇIOGLU, Omur Y. What determines user satisfaction in ERP projects: benefits, barriers or risks? Journal of Enterprise Information Management. v.22, n.6, p. 690-708, 2009.

SILVEIRA, M. A. P. ; ZWICKER, R. A tecnologia de informação como fonte de competitividade e como apoio para a formação de arranjos produtivos locais: um estudo nas principais cadeias produtivas da Região do ABC paulista. In: Encontro da ANPAD: Anais, 2004.

SANTOS, Glauber Eduardo de Oliveira. Cálculo Amostral: Calculadora on-line. Disponível em: <http://www.calculoamostral.vai.la> Acesso em Jan. 2012.

STERNAD, Simona; GRADISAR, Miro; BOBEK, Samo. The influence of external factors on routine ERP usage. Industrial Management \& Data Systems. v.111, n.9, p. 1511-1530, 2011.

STRAKA, Kristen L. Wireless telephone systems and the impact on patient satisfaction scoring: a pilot study. Journal of Pediatric Nursing. v. 25, n. 1, p. 33-34, 2010.

TORKZADEH, Gholamreza; DOLL, William, J. The development of a tool for measuring the perceived impact of information technology on work. Omega, New York, v. 27, p. 327-339, 1999.

VERGARA, S.C. Projetos e Relatórios de Pesquisa em Administração. São Paulo: Atlas,2006.

WU, Jen-Her; WANG, Yuh-Min; CHIEN, Mien-Chih Chang; TAI, Wei-Chun. An examination of ERP user satisfaction in Taiwan. In: International Conference on System Sciences, 35, 2002, Hawaii: HICSS, 2002. 
WU, Jen-Her; WANG, Yu-Min. Measuring ERP success: the key-users viewpoint of to erp to produce a viable is in the organization. Computers in Human Behavior. Japan, v.23, n. 3, p. 1582-1596, May, 2007.

WU, Jen-Her; WANG, Yu-Min. Measuring ERP success: the ultimate user's view. International Journal of Operations \& Production Management. United Kingdom, v.26, n. 8, p. 882-903, 2006.

ZWASS, Vladimir. Management Information Systems. EUA:WCB, 1992. 